\title{
Colonização de macroinvertebrados bentônicos em detritos foliares em um riacho de primeira ordem na Floresta Atlântica do nordeste brasileiro
}

\author{
Iris Gabrielly Arruda dos Santos \& Gilberto Gonçalves Rodrigues
}

Laboratório de Avaliação, Recuperação e Restauração de Ecossistemas - ARRE Água, Departamento de Zoologia, Programa de Pós-Graduação em Biologia Animal, Centro de
Ciências Biológicas, Universidade Federal de Pernambuco, Av. da Engenharia - Cidade Universitária, 50740-600 Recife, PE. (iris.arrudasantos@gmail.com; gillberto.rodrigues@ufpe.br)

\begin{abstract}
Colonization of leaf litter by benthic macroinvertebrates in an Atlantic forest stream from northeast Brazil. The aim of this study was to analyze the colonization of Inga ingoides (Leguminosae) leaves by benthic macroinvertebrates in field experiments. A total of 270 leaf packs, distributed in three stretches, were submerged of similar morphology and physical and chemical parameters of water between February to July 2013, and retrieved after 7, 30, 60, 90 and 120 days. Two treatments were used: colonization and exclusion of benthic macroinvertebrates. The leaf weight loss (\%R) was greater to colonization than exclusion of benthic fauna, showing the role of macroinvertebrates in the leaf breakdown. Shredders played a minor role in the leaf breakdown. Filtering was the main trophic feeding group (45.6\%), followed by predator (31.2\%), collector (11.8\%), scrapers (6.7\%) and shredders (4.8\%). Triplectides (Trichoptera: Leptoceridae), Polypedillum, and Stenochironomus (Diptera, Chironomidae) were the most frequent shredders.
\end{abstract}

KEYWORDS. Benthic fauna, leaf breakdown, protected areas, shredders, stream ecology.

RESUMO. O objetivo do estudo foi avaliar a colonização de macroinvertebrados bentônicos em detritos foliares de Inga ingoides (Leguminosae) em experimentos de campo em um riacho de primeira ordem da Mata Atlântica do Estado de Pernambuco no nordeste do Brasil. Um total de 270 bolsas de folhiço, distribuídas em três trechos do riacho com características similares em relação à morfologia e parâmetros físicos e químicos da água foram submersas, entre fevereiro a julho de 2013 e retiradas após 7, 30, 60, 90 e 120 dias. Dois tratamentos foram utilizados: colonização e exclusão da macrofauna. O percentual de biomassa remanescente final (\%R) foi maior para bolsas de colonização do que para as de exclusão, evidenciando a efetiva participação dos macroinvertebrados no processo de decomposição. Os fragmentadores apresentaram menor participação no processo de decomposição foliar de I. ingoides, sendo Tripletides (Trichoptera: Leptoceridae), Polypedillum e Stenochironomus (Diptera, Chironomidae) os táxons predominantes na macrofauna. Filtrador foi o grupo trófico funcional predominante (45,6\%), seguido por predador $(31,2 \%)$, coletor $(11,8 \%)$, raspador $(6,7 \%)$ e fragmentador $(4,8 \%)$.

PALAVRAS-CHAVE. Decomposição foliar, ecologia de riachos, fauna bentônica, fragmentadores, unidade de conservação.

Os macroinvertebrados são importantes para os ecossistemas aquáticos por desempenhar papel fundamental na degradação do material alóctone em riachos, principalmente nas nascentes. São comumente utilizados como ferramenta em Unidades de Conservação, para o monitoramento da qualidade da água, auxiliando na gestão das águas dessas áreas protegidas que atuam como prestadora de serviços ambientais. Assim, os macroinvertebrados podem se configurar como complemento essencial nas análises de água, contribuindo para a avaliação ecológica desses ecossistemas.

A decomposição nos corpos d'água como processo ecossistêmico é tão importante como a produção, pois libera energia e nutrientes no sistema e, portanto, influencia as variáveis físicas, químicas e biológicas, podendo também ser influenciada por essas, servindo inclusive como índice da qualidade ambiental. A decomposição torna-se mais rápida quando se tem uma densidade elevada de macroinvertebrados (GRAÇA et al., 2005).

Mecanismos físicos são importantes na aceleração da decomposição, como a lixiviação ou quebra de detritos (Benfield et al., 1979; CAstillo, 2009). Outro modo de fragmentação é o processo realizado pelos invertebrados, principalmente, os que ocupam o nível trófico dos herbívoros fragmentadores, que colonizam e se alimentam dos detritos foliares já colonizados ou não por microrganismos e contribuem para decomposição dos riachos (WEBSTER \& BENFIELD, 1986). Os fragmentadores são escassos nos trópicos devido às altas temperaturas, sua diversidade torna-se mais baixa, aumentando a diversidade dos demais grupos tróficos (BOYero et al., 2011). Em uma escala global esses autores ressaltaram que os padrões latitudinais da diversidade dos fragmentadores ocorrem em forma inversa aos demais grupos, diminuindo em relação aos trópicos. Baixas temperaturas e alta qualidade das folhas, em termos de nitrogênio e fósforo nas folhas, favorecem a presença de fragmentadores nas regiões temperadas. A baixa frequência de fragmentadores em regiões tropicais pode estar associada às altas temperaturas e baixa qualidade nutricional das folhas (BOYERo et al., 2011). Devido a essas questões, relativas à importância dos macroinvertebrados no processo de decomposição, é que esse estudo procura elucidar a importância dos fragmentadores no processo de decomposição de detritos foliares em riachos de $1^{\mathrm{a}}$ ordem, em uma área protegida de Mata Atlântica inserida em uma unidade de conservação de proteção integral, que fornece serviços ecossistêmicos e ambientais relevantes para a Mata Sul do Estado de Pernambuco. 
No Estado de Pernambuco não há estudos sobre macroinvertebrados bentônicos em riachos voltados para análise da estrutura e distribuição desses (CuNHA et al., 2014). Na Mata Atlântica de Pernambuco, historicamente, grandes áreas foram convertidas em monoculturas de cana-de-açúcar, sendo esses pequenos fragmentos de mata importantes para a manutenção dos cursos d'água. Estudos sobre processos ecossistêmicos, como colonização e decomposição foliar, são ainda incipientes no Estado e no nordeste. Esse estudo contempla a colonização por invertebrados bentônicos em detritos foliares e sua relação com a decomposição em riacho de $1^{\text {a }}$ ordem uma Reserva Biológica na Mata Atlântica do nordeste brasileiro, sendo considerado pioneiro nessa abordagem.

\section{MATERIAIS E MÉTODOS}

Área de Estudo. A Reserva Biológica de Saltinho (REBIO Saltinho), localizada no município de Tamandaré, litoral sul de Pernambuco $\left(08^{\circ} 44^{\prime} 13-08^{\circ} 43^{\prime}\right.$ 'S; 35 $35^{\circ} 10^{\prime}$ $35^{\circ} 11^{\prime} \mathrm{W}$ ) foi criada pelo Decreto $\mathrm{n}^{\circ} 88.744 / 1983$ e se enquadra no SNUC como Unidade de Conservação de Proteção Integral (CunHa et al., 2014). O clima predominante da região é do tipo tropical úmido (As') segundo a classificação de Köppen. A temperatura média anual varia entre $22^{\circ} \mathrm{C}$ e $26^{\circ} \mathrm{C}$ (CUnHA et al., 2014).

A Bacia Hidrográfica do Córrego do Saltinho integra aproximadamente 22.634 ha e apresenta inúmeras nascentes e um córrego principal que dá o nome à REBIO Saltinho, o riacho Saltinho. O riacho nasce fora da área da REBIO Saltinho e apresenta seu fluxo principal de noroeste para sudoeste, atravessando a REBIO Saltinho e desaguando no Rio Mamucabas, próximo à foz, no Oceano Atlântico (CunHA et al., 2014). Na área da REBIO Saltinho, a bacia abrange área de 475 ha, constituindo a totalidade da REBIO Saltinho. A montante da sede principal encontram-se dois reservatórios que servem de abastecimento para a cidade de Tamandaré e, a jusante, uma queda d'água de aproximadamente $10 \mathrm{~m}$ denominada Bulha D'água.

Procedimentos metodológicos. $\mathrm{O}$ riacho foi dividido em três trechos de coleta (A, B, C). O bloco A, constituído de um afluente que deságua no riacho Saltinho, está localizado a oeste do canal principal. Os blocos B e C localizam-se no trecho principal, distante cerca de $150 \mathrm{~m}$ entre si. Desta forma, as unidades amostrais foram independentes e com características físicas similares. Cada trecho apresenta $150 \mathrm{~m}$ de extensão, totalizando $450 \mathrm{~m}$ de riacho amostrado. Os trechos foram subdivididos em nove pontos de coleta, onde foram submersas cinco bolsas de colonização (Tratamento 1: $>10 \mathrm{~mm}$ de malha) e cinco de exclusão da macrofauna (Tratamento 2: 0,045 mm de malha) em cada ponto de coleta, totalizando 270 unidades amostrais. Foi realizada a morfometria do riacho visando sua caracterização para que os experimentos fossem conduzidos em trechos similares, evitando disparidades entre os ambientes, a profundidade média foi de 19,4 $( \pm 10,64) \mathrm{cm}$, a vazão média (variação da coluna d'água) foi $0,96( \pm 0,47) \mathrm{cm}$, a altura do barranco (declive) foi 78,7 $( \pm 44,8) \mathrm{cm}$ e a largura do riacho foi $224,7( \pm 94,9) \mathrm{cm}$. Os estudos foram conduzidos no período de fevereiro a julho de 2013, com coletas mensais.

As bolsas de folhiço de malha maior foram confeccionados com saco de polietileno high-density (HDPE/PP) e as bolsas de malha menor com tecido de voil. Em cada bolsa foram adicionadas $4,0 \mathrm{~g}$ de folhas de Inga ingoides (Rich) Willd (Leguminosae). As folhas foram coletadas de um único indivíduo na área de estudo, desidratadas em temperatura ambiente e após secas em estufa a $60^{\circ} \mathrm{C}$ por $48 \mathrm{~h}$. As bolsas foram fixadas nas margens do riacho por fio de nylon e retiradas após $24 \mathrm{~h}$ (determinação do peso inicial), 7, 30, 60, 90 e 120 dias de exposição. A escolha pela utilização de $I$. ingoides foi devida à alta ocorrência, frequência e disponibilidade na mata ciliar do riacho Saltinho.

Em laboratório as bolsas de decomposição foram abertas e o material foliar lavado em água corrente em peneiras de malha de $200 \mu \mathrm{m}$ de abertura. O material orgânico particulado bruto (MOPB $>1,0 \mathrm{~mm}$ ) foi separado e tanto os organismos retidos na peneira e o material orgânico particulado fino (MOPF $<1,0 \mathrm{~mm}$ ) foram fixados em álcool $70 \%$ para posterior triagem, separação e identificação dos macroinvertebrados. Os organismos foram separados e quantificados por grupos taxonômicos (menor nível possível para cada grupo) e a identificação dos exemplares realizada com consulta à bibliografia (e.g., Domínguez et al., 2006; Borkent \& Spinelli, 2007; PAssos et al., 2007; Domínguez \& Fernandéz, 2009; Epler, 2010; Mugnai et al., 2010; Trivinho-Strixino, 2011; Hamada et al., 2014) e com a confirmação de especialistas, quando possível. O material está depositado na coleção do Laboratório ARRE Água/UFPE.

Os detritos foliares (MOPB $>1,0 \mathrm{~mm}$ ) sem macroinvertebrados foram secos em estufa a $60^{\circ} \mathrm{C}$ até atingir peso constante e pesados em balança analítica $(0,0001 \mathrm{~g})$ para determinação da perda de massa foliar. Foi determinada a biomassa remanescente final (\%R) e a partir desta foi calculada a taxa de decomposição $(-k)$. Para esta análise foi utilizado um modelo exponencial (Petersen \& Cummins, 1974; Anderson et al., 1983; Webster \& Benfield, 1986; Benfield, 1996) e utilizado sistematicamente (e.g., Graça et al., 2005; Barbosa \& Rodrigues, 2007; Ruppenthal et al., 2007; Agra et al., 2013). Foi aplicada a correlação de Spearman (S) para analisar a perda da massa foliar e abundância dos macroinvertebrados, para (i) os dois tipos de tratamento, (ii) tempo de exposição e (iii) os blocos de coleta, utilizando o software BioEstat 5.0.

Para a análise da estrutura da comunidade de macroinvertebrados foram utilizados os índices de riqueza, abundância, constância e dominância. Para riqueza de táxon foi construído curvas de rarefação a partir da colonização e exclusão de macroinvertebrados em todos os blocos e tempo de exposição. Para isto utilizou-se o pacote estatístico PAST ${ }^{\circledR}$ 2.17. Os táxons foram classificados 
como Constantes quando apresentaram $>50 \%$ das coletas, Acessórios $<25 \%$ a $50 \%$ das coletas e Acidentais com $<25 \%$ das coletas (BoDENHEIMER, 1938). A dominância foi calculada tomando como base o índice de Simpson (D), que considera a riqueza, abundância e a porcentagem de ocorrência de cada táxon (BROWER \& ZARR, 1984).

Para avaliação dos grupos tróficos funcionais (GTF) de quais macroinvertebrados participam efetivamente da decomposição, os organismos foram classificados conforme seus hábitos alimentares de acordo com o nível trófico e/ou os mecanismos/hábitos alimentares de cada táxon (Cummins, 1973; MerRitT et al., 1984): filtradores, raspadores, fragmentadores, coletores e predadores.

\section{RESULTADOS}

O experimento de colonização e decomposição de folhas de I. ingoides por macroinvertebrados bentônicos durou 120 dias. $\mathrm{O}$ tratamento 1 obteve maior perda de massa foliar, ficando em torno de $10 \%$, enquanto que o tratamento 2 apresentou cerca de $40 \%$ de biomassa remanescente $(\% \mathrm{R})$ (Fig. 1). Dos três trechos, os que obtiveram maiores $\% \mathrm{R}$ foram o bloco B (3\%) e o bloco A (10\%) enquanto que o bloco C, com $25 \%$, apresentou menor $\% \mathrm{R}$ aos 120 dias no tratamento 1 (Fig. 2); no tratamento 2 , o \% R foi menor que $55 \%$ em todos os blocos (Fig. 3). A velocidade da taxa de decomposição $(-k)$ foi considerada lenta para o tratamento 2 e média para o tratamento 1 (Tab. I).

A análise de Spearman (S) mostrou que existe correlação positiva $(p=0,001)$ entre o tipo de malha, e negativa entre o tempo de exposição da bolsa e os trechos do riacho (blocos) (Tab. II). Ao aplicarmos a mesma análise para a abundância dos organismos, verificamos

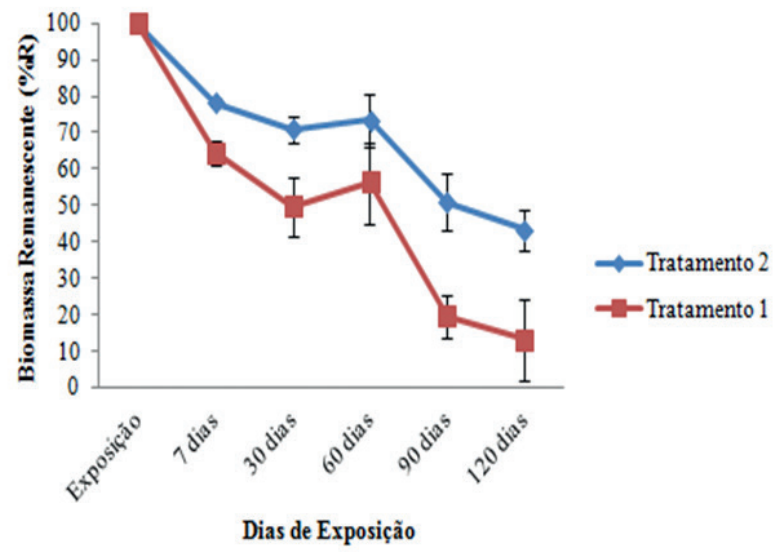

Fig. 1. Comparação do percentual médio de Biomassa Remanescente $(\% \mathrm{R})$ para litterbags dos dois tratamentos (tratamento 1: malha $>10 \mathrm{~mm}$ e tratamento 2: malha $<0,045 \mathrm{~mm}$ ) em três trechos (A, B, C) em todos os períodos de coleta (fevereiro a junho de 2013) do riacho Saltinho, Pernambuco, Brasil.

que a mesma está correlacionada negativamente com o tratamento e o tempo de exposição, e positivamente com o bloco de coleta (Tab. II). Os resultados mostraram que a perda de massa foliar apresenta diferença significativa para os dois tipos de malhas $(\mathrm{S}=0,3486 ; \mathrm{p}>0,001)$. Da mesma forma, se verificou diferenças entre o tempo de exposição (dias) e os blocos. A abundância dos organismos por sua vez foi significativa para os tratamentos (tipo de malha) e para o tempo de exposição (dias).

A abundância total dos macroinvertebrados foi de 11.740 indivíduos para o trecho B, 10.129 para o trecho $\mathrm{A}$ e 9.573 para o bloco $\mathrm{C}$, totalizando 31.442 indivíduos. $\mathrm{O}$ trecho C apresentou uma densidade com $0,67 \pm 2,40$ ind.g-1, seguido por B com $0,59 \pm 1,74$, e por A com $0,10 \pm 0,18$ para o tratamento 1 . No tratamento 2 esse padrão se repete,
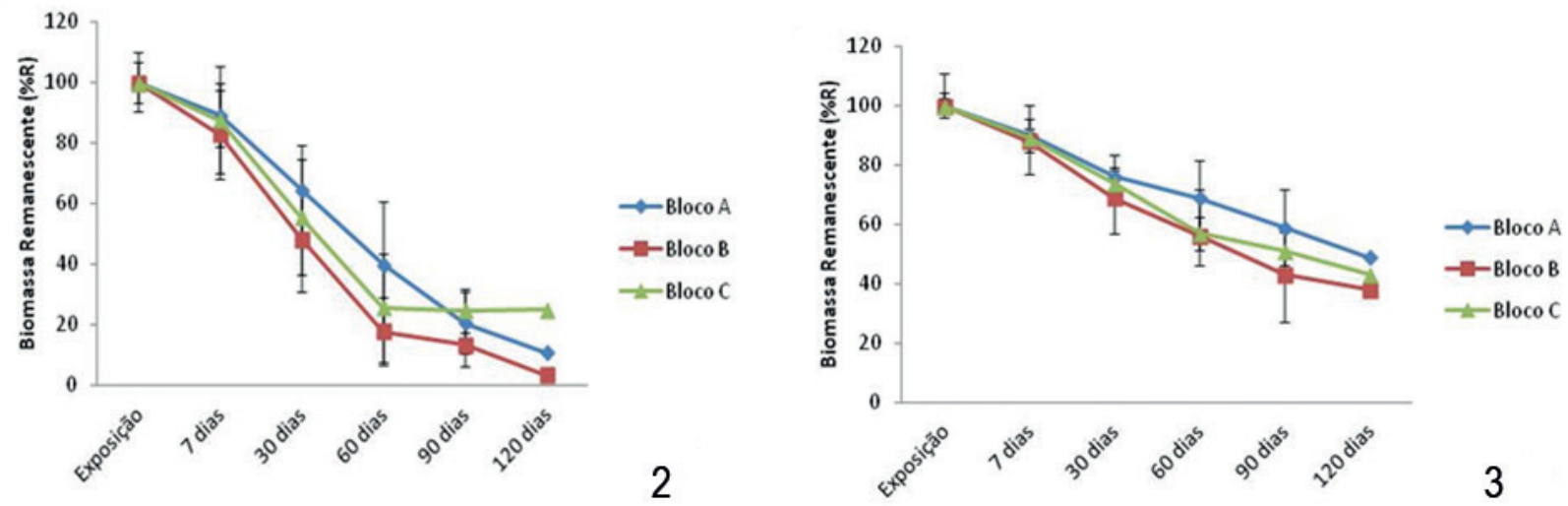

Figs 2, 3. Comparação do percentual médio de Biomassa Remanescente (\%R) das bolsas de colonização e exclusão da macrofauna expostas nos blocos de coleta (A, B, C) em todos os períodos de tempo (7, 30, 60, 90 e 120 dias), no riacho Saltinho, na Reserva Biológica de Saltinho, Pernambuco, Brasil. Fig. 2, tratamento 1: malha $>10,0 \mathrm{~mm}$; Fig. 3, tratamento 2: malha $<0,045 \mathrm{~mm}$.

Tab. I. Média final da taxa de decomposição (-k) para Inga ingoides para todos os períodos (7, 30, 60, 90, 120 dias) e trechos do riacho Saltinho, Pernambuco, Brasil (blocos A, B, C) (*, decomposição lenta; **, decomposição média).

\begin{tabular}{ccccc}
\hline & Tratamento 1 & & Tratamento 2 \\
\hline Bloco A & Bloco B & Bloco C & Bloco A & Bloco B \\
$-k=0,006^{* *}$ & $-k=0,007^{* *}$ & $-k=0,006^{* *}$ & $-k=0,0008^{*}$ & $-k=0,0006^{*}$ \\
\hline
\end{tabular}


Tab. II. Correlação de Spearman (***p>0,001) para as análises de biomassa remanescente $(\% \mathrm{R})$ e abundância de macroinvertebrados bentônicos presentes nas bolsas de folhiço expostas no riacho Saltinho, de fevereiro a julho de 2013, na Reserva Biológica de Saltinho, Pernambuco, Brasil.

\begin{tabular}{lcc}
\hline & Biomassa (\%R) & Abundância fauna \\
\hline Tratamento (tipo de malha) & $0,3486^{* * *}$ & $-0,2989$ \\
Tempo (exposição das bolsas) & $-0,7820$ & $-0,5157$ \\
Bloco (trechos A,B,C) & $-0,0464$ & $0,1436^{* * *}$
\end{tabular}

porém com densidades menores. O trecho $\mathrm{C}$ apresentou $0,09 \pm 0,39$, B $0,05 \pm 0,13$ e A $0,04 \pm 0,11$ ind. $\mathrm{g}^{-1}$.

A riqueza foi maior para o tratamento 1 , como demonstrado na análise de rarefação (Fig. 4). A maioria dos táxons do tratamento 2 está presente no tratamento 1, porém com tamanho diminuto (estágios iniciais). $\mathrm{O}$ tratamento 1 estabilizou aos 40 dias de exposição, enquanto o tratamento 2, mesmo após 120 dias, não ocorreu saturação, devido à presença de cerca de $40 \% \mathrm{R}$.

A abundância total dos macroinvertebrados bentônicos no tratamento 1 foi representada por DipteraChironomidae, Crustacea-Ostracoda, Acari-Hydracarina, Coleoptera (larvas e adultos), Ephemeroptera, Crustacea-

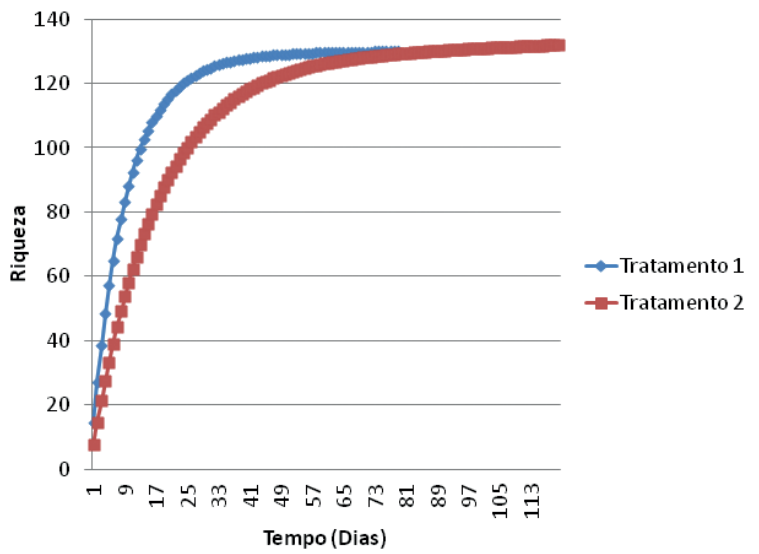

Fig. 4. Curva de rarefação das morfoespécies de invertebrados bentônicos em bolsas de folhiço expostas de fevereiro a julho de 2013 nos três blocos de coleta (A, B, C) e em todos os períodos de tempo $(7,30,60$, 90 e 120 dias) no riacho Saltinho, na Reserva Biológica de Saltinho, Pernambuco, Brasil.
Cladocera, Annelida-Oligochaeta, Trichoptera, MolluscaGastropoda, e Diptera-Ceratopogonidae.

A maioria dos organismos foram considerados não dominantes $(50,4 \%)$ em relação à dominância $(42,6 \%)$ no tratamento 1 . O tratamento 2 apresentou organismos não dominantes $(40,0 \%)$ e dominantes $(21,7 \%)$, sendo que $38,3 \%$ dos organismos que foram encontrados no tratamento 1 estiveram ausentes no tratamento 2 (Tab. III). Quanto a constância, 73,0\% foram considerados acidentais no tratamento 1 e somente $13,0 \%$ constantes. No tratamento 2 a maioria dos organismos foram acidentais $(43,5 \%)$ e $7,8 \%$ constantes. Os organismos da meiofauna foram constantes em ambos os tratamentos, sendo que os da macrofauna (Bezzia, Caenis, Farrodes) apresentam tamanho diminuto (Tab. III).

Ao verificar a sucessão dos macroinvertebrados no tratamento 1, foi visto que, no geral, existe a predominância dos organismos filtradores, seguindo por predadores, coletores, raspadores e fragmentadores em todos os períodos de coleta (Fig. 5). Para tratamento 2 foi verificado a predominância dos filtradores em todos os períodos de tempo, devido principalmente a presença de Ostracoda (Fig. 6). Filtrador foi o grupo mais representativo em comparação aos demais, com 45,6\% da abundância total, seguido pelos predadores, perfazendo $32 \%$ do total, coletores em torno de $10 \%$ e raspadores e fragmentadores com valor menor que $10 \%$ (Tab. IV). O tratamento 2 apresentou o mesmo padrão de GTF: filtrador seguido por predador seguido por coletor seguido por raspador seguido por fragmentador.

Em relação aos fragmentadores, 310 organismos foram encontrados, sendo a maioria Triplectides
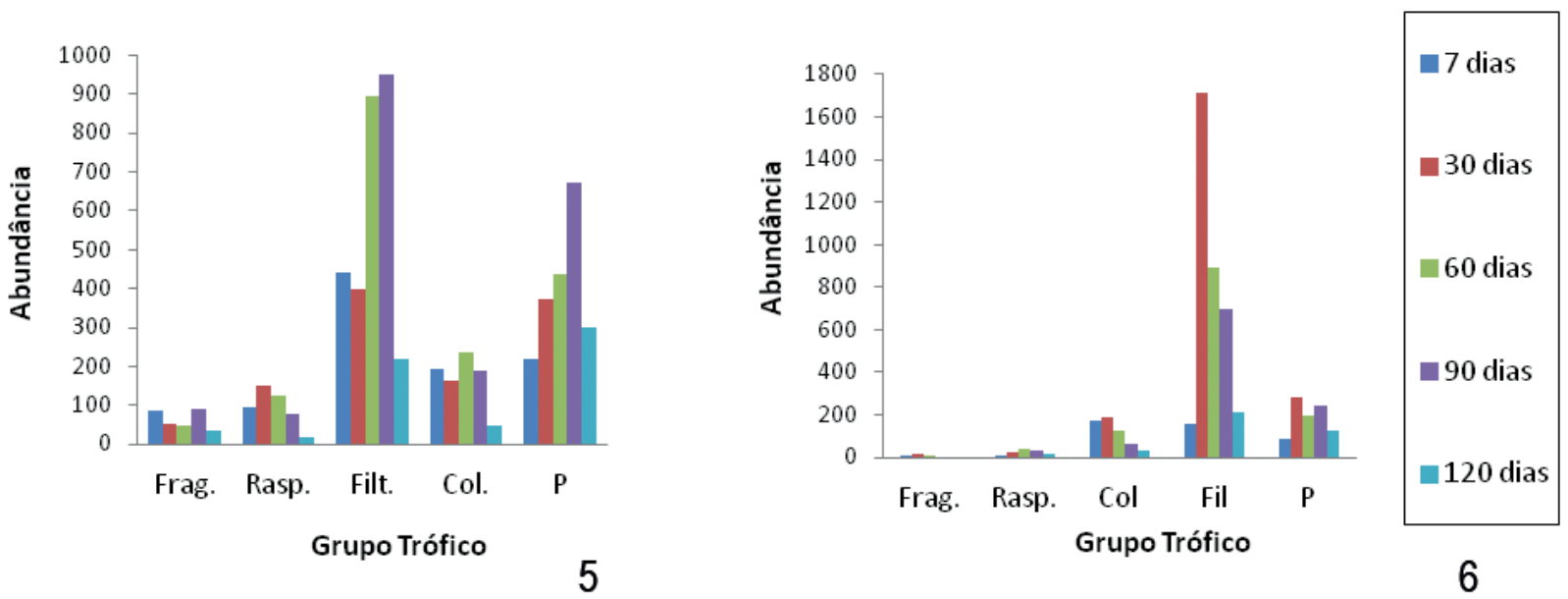

Figs 5, 6. Sucessão da colonização dos macroinvertebrados bentônicos em detritos foliares de Inga ingoides em bolsas de folhiço expostas nos trechos A, B e C do riacho Saltinho, na Reserva Biológica de Saltinho, Pernambuco, Brasil. Fig. 5, tratamento 1: malha $>10$ mm; Fig. 6, tratamento 2: malha $<0,045 \mathrm{~mm}$. 
Tab. III. Composição de táxons de macroinvertebrados bentônicos para os tratamentos 1 e 2 com os respectivos Grupos Tróficos Funcionais (GTF) (C/F, Coletor/Filtrador; F/F, Filtrador/Fitófago; F, Fragmentador; C/C, Coletor/Catador; P, Predador; R, Raspador) e análise de Dominância (Dom) (D, Dominante; N/D, Não Dominante) de Constância (Const) (Acd, Acidental; Acs, Acessório e C, Constante) (-, Ausente; *, Meiofauna). As bolsas foram expostas de fevereiro a julho de 2013 no riacho Saltinho, na Reserva Biológica de Saltinho, Pernambuco.

\begin{tabular}{|c|c|c|c|c|c|}
\hline \multirow{2}{*}{$\begin{array}{l}\text { Composição de táxons } \\
\text { Invertebrados bentônicos }\end{array}$} & \multirow[b]{2}{*}{ GTF } & \multicolumn{2}{|c|}{ Tratamento 1} & \multicolumn{2}{|c|}{ Tratamento 2} \\
\hline & & Const & Dom & Const & Dom \\
\hline \multicolumn{6}{|l|}{ Insecta, Coleoptera, Elmidae } \\
\hline Mycrocylloepus & $\mathrm{R}$ & Acs & $\mathrm{D}$ & Acd & $\mathrm{N} / \mathrm{D}$ \\
\hline Noelmis & $\mathrm{R}$ & Acd & $\mathrm{N} / \mathrm{D}$ & & $\mathrm{D}$ \\
\hline Xenelmis & $\mathrm{P}$ & Acd & $\mathrm{N} / \mathrm{D}$ & Acd & $\mathrm{N} / \mathrm{D}$ \\
\hline \multicolumn{6}{|c|}{ Insecta, Coleoptera, Hydrophilidae } \\
\hline Anacaena & $\mathrm{P}$ & Acd & $\mathrm{N} / \mathrm{D}$ & - & $\mathrm{N} / \mathrm{D}$ \\
\hline Derallus & $\mathrm{P}$ & Acd & $\mathrm{N} / \mathrm{D}$ & - & $\mathrm{N} / \mathrm{D}$ \\
\hline Enochrus & $\mathrm{P}$ & Acd & $\mathrm{N} / \mathrm{D}$ & - & $\mathrm{N} / \mathrm{D}$ \\
\hline Helochares & $\mathrm{P}$ & Acd & $\mathrm{N} / \mathrm{D}$ & - & $\mathrm{N} / \mathrm{D}$ \\
\hline Paracymus & $\mathrm{C} / \mathrm{C}$ & Acd & $\mathrm{N} / \mathrm{D}$ & Acd & $\mathrm{N} / \mathrm{D}$ \\
\hline \multicolumn{6}{|c|}{ Insecta, Coleoptera, Noteridae } \\
\hline Suphis & $\mathrm{P}$ & Acd & $\mathrm{N} / \mathrm{D}$ & Acd & $\mathrm{N} / \mathrm{D}$ \\
\hline \multicolumn{6}{|c|}{ Insecta, Diptera, Ceratopogonidae } \\
\hline Bezzia & $\mathrm{P}$ & $\mathrm{C}$ & $\mathrm{D}$ & $\mathrm{C}$ & $\mathrm{D}$ \\
\hline Culicoides & $\mathrm{P}$ & Acd & $\mathrm{D}$ & Acd & $\mathrm{D}$ \\
\hline Leptoconops & $\mathrm{C} / \mathrm{C}$ & Acd & $\mathrm{N} / \mathrm{D}$ & Acd & $\mathrm{N} / \mathrm{D}$ \\
\hline \multicolumn{6}{|c|}{ Insecta, Diptera, Chironomidae } \\
\hline Chironomus & $\mathrm{C} / \mathrm{C}$ & Acd & $\mathrm{D}$ & Acd & $\mathrm{N} / \mathrm{D}$ \\
\hline Cladopelma & $\mathrm{C} / \mathrm{C}$ & - & $\mathrm{N} / \mathrm{D}$ & Acd & $\mathrm{N} / \mathrm{D}$ \\
\hline Dicrotendipes & $\mathrm{C} / \mathrm{C}$ & Acd & $\mathrm{N} / \mathrm{D}$ & Acd & $\mathrm{N} / \mathrm{D}$ \\
\hline Djalmabatista & $\mathrm{P}$ & Acd & $\mathrm{N} / \mathrm{D}$ & - & - \\
\hline Endotribelos & $P$ & Acd & $\mathrm{N} / \mathrm{D}$ & - & - \\
\hline Fittkauimyia & $\mathrm{P}$ & - & - & Acd & $\mathrm{N} / \mathrm{D}$ \\
\hline Goeldichironomus & $\mathrm{C} / \mathrm{C}$ & Acd & $\mathrm{N} / \mathrm{D}$ & Acd & $\mathrm{N} / \mathrm{D}$ \\
\hline Harnischia & $\mathrm{C} / \mathrm{F}$ & Acd & $\mathrm{D}$ & - & $\mathrm{N} / \mathrm{D}$ \\
\hline Hudsonimyia & $\mathrm{P}$ & Acd & $\mathrm{N} / \mathrm{D}$ & Acd & $\mathrm{N} / \mathrm{D}$ \\
\hline Labrundinia & $\mathrm{P}$ & Acd & $\mathrm{N} / \mathrm{D}$ & - & - \\
\hline Larsia & $\mathrm{P}$ & Acd & $\mathrm{D}$ & Acd & $\mathrm{D}$ \\
\hline Microtanytarsus & $\mathrm{C} / \mathrm{F}$ & Acd & $\mathrm{N} / \mathrm{D}$ & - & - \\
\hline Nilotanypus & $\mathrm{P}$ & Acd & $\mathrm{N} / \mathrm{D}$ & Acd & $\mathrm{N} / \mathrm{D}$ \\
\hline Oukuriella & $\mathrm{C} / \mathrm{C}$ & Acd & $\mathrm{D}$ & Acd & $\mathrm{N} / \mathrm{D}$ \\
\hline Parachironomus & $\mathrm{P}$ & Acd & $\mathrm{N} / \mathrm{D}$ & - & - \\
\hline Paramerina & $\mathrm{P}$ & Acd & $\mathrm{N} / \mathrm{D}$ & - & - \\
\hline Parakiefferiella & $\mathrm{C} / \mathrm{C}$ & Acd & $\mathrm{N} / \mathrm{D}$ & - & - \\
\hline Paratanytarsus & $\mathrm{C} / \mathrm{F}$ & Acd & $\mathrm{D}$ & Acd & $\mathrm{D}$ \\
\hline Paratendipes & $\mathrm{C} / \mathrm{C}$ & Acd & $\mathrm{N} / \mathrm{D}$ & Acd & $\mathrm{N} / \mathrm{D}$ \\
\hline Parapentaneura & $\mathrm{P}$ & Acd & $\mathrm{N} / \mathrm{D}$ & - & - \\
\hline Phaenopsectra & $\mathrm{C} / \mathrm{C}$ & - & - & Acd & $\mathrm{N} / \mathrm{D}$ \\
\hline Polypedillum & $\mathrm{F}$ & Acd & $\mathrm{D}$ & Acd & $\mathrm{N} / \mathrm{D}$ \\
\hline Procladius & $\mathrm{P}$ & - & - & Acd & $\mathrm{N} / \mathrm{D}$ \\
\hline Riethia & $\mathrm{C} / \mathrm{C}$ & Acd & $\mathrm{N} / \mathrm{D}$ & - & - \\
\hline Stempellinella & $\mathrm{P}$ & Acd & $\mathrm{N} / \mathrm{D}$ & - & - \\
\hline Stenochironomus & $\mathrm{F}$ & Acd & $\mathrm{N} / \mathrm{D}$ & Acd & $\mathrm{D}$ \\
\hline Stictochironomus & $\mathrm{C} / \mathrm{C}$ & Acd & $\mathrm{N} / \mathrm{D}$ & - & - \\
\hline Tanytarsus & $\mathrm{C} / \mathrm{F}$ & Acs & $\mathrm{D}$ & Acd & $\mathrm{D}$ \\
\hline Xestochironomus & $\mathrm{C} / \mathrm{C}$ & - & - & Acd & N/D \\
\hline Zaverlimyia & $\mathrm{P}$ & $\mathrm{C}$ & $\mathrm{D}$ & Acd & $\mathrm{D}$ \\
\hline \multicolumn{6}{|l|}{ Insecta, Diptera, Culicidae } \\
\hline Sabethini & $\mathrm{C} / \mathrm{C}$ & Acd & $\mathrm{N} / \mathrm{D}$ & - & - \\
\hline \multicolumn{6}{|l|}{ Insecta, Diptera, Dixidae } \\
\hline Dixella & $\mathrm{F}$ & Acd & $\mathrm{N} / \mathrm{D}$ & - & - \\
\hline \multicolumn{6}{|l|}{ Insecta, Diptera, Simuliidae } \\
\hline Prosomulium & $\mathrm{P}$ & Acd & $\mathrm{N} / \mathrm{D}$ & - & - \\
\hline \multicolumn{6}{|l|}{ Insecta, Diptera, Syrphidae } \\
\hline Eritalis & $\mathrm{P}$ & Acd & $\mathrm{D}$ & Acs & $\mathrm{D}$ \\
\hline Toxomerus & $\mathrm{P}$ & Acd & $\mathrm{N} / \mathrm{D}$ & Acd & $\mathrm{D}$ \\
\hline \multicolumn{6}{|l|}{ Insecta, Diptera, Tabanidae } \\
\hline Chrysops & $\mathrm{P}$ & Acd & $\mathrm{D}$ & Acd & $\mathrm{N} / \mathrm{D}$ \\
\hline Insecta, Diptera, Tipulidae & & & & & \\
\hline Dolichopeza & $\mathrm{P}$ & Acd & $\mathrm{N} / \mathrm{D}$ & - & - \\
\hline Tipula & $\mathrm{C} / \mathrm{C}$ & Acd & $\mathrm{N} / \mathrm{D}$ & - & - \\
\hline Insecta, Ephemeroptera, B & & & & & \\
\hline Wantzoyphius & $\mathrm{F}$ & Acd & $\mathrm{N} / \mathrm{D}$ & - & - \\
\hline
\end{tabular}


Tab. III. Cont

\begin{tabular}{|c|c|c|c|c|c|}
\hline \multirow{2}{*}{$\begin{array}{l}\text { Composição de táxons } \\
\text { Invertebrados bentônicos }\end{array}$} & \multirow[b]{2}{*}{ GTF } & \multicolumn{2}{|c|}{ Tratamento 1} & \multicolumn{2}{|c|}{ Tratamento 2} \\
\hline & & Const & Dom & Const & Dom \\
\hline \multicolumn{6}{|l|}{ Insecta, Ephemeroptera, Caenidae } \\
\hline Caenis & $\mathrm{C} / \mathrm{C}$ & $\mathrm{C}$ & $\mathrm{D}$ & $\mathrm{C}$ & $\mathrm{N} / \mathrm{D}$ \\
\hline \multicolumn{6}{|l|}{ Insecta, Ephemeroptera, Leptophlebiidae } \\
\hline Farrodes & $\mathrm{C} / \mathrm{F}$ & $\mathrm{C}$ & $\mathrm{D}$ & $\mathrm{C}$ & $\mathrm{N} / \mathrm{D}$ \\
\hline Hydrosmilodon & $\mathrm{R}$ & - & - & Acd & $\mathrm{D}$ \\
\hline Massartela & $\mathrm{F}$ & Acd & $\mathrm{N} / \mathrm{D}$ & - & - \\
\hline Miroculis & $\mathrm{R}$ & $\mathrm{C}$ & $\mathrm{D}$ & - & - \\
\hline Simothraulopsis & $\mathrm{R}$ & Acd & $\mathrm{N} / \mathrm{D}$ & Acd & $\mathrm{D}$ \\
\hline Ulmeritoides & $\mathrm{R}$ & Acd & $\mathrm{N} / \mathrm{D}$ & - & - \\
\hline \multicolumn{6}{|l|}{ Insecta, Ephemeroptera, Leptohyphidae } \\
\hline Leptohyphes & $\mathrm{C} / \mathrm{C}$ & $\mathrm{C}$ & $\mathrm{D}$ & Acd & $\mathrm{D}$ \\
\hline \multicolumn{6}{|l|}{ Insecta, Lepidoptera } \\
\hline Arctiidae sp. 1 & $\mathrm{~F}$ & Acd & $\mathrm{N} / \mathrm{D}$ & - & - \\
\hline Crambidae Parapoynx & $\mathrm{F}$ & Acd & $\mathrm{N} / \mathrm{D}$ & - & - \\
\hline Epipyropidae sp. 1 & $\mathrm{~F}$ & - & - & Acd & $\mathrm{N} / \mathrm{D}$ \\
\hline Noctuidae sp. 1 & $\mathrm{~F}$ & Acd & $\mathrm{N} / \mathrm{D}$ & - & - \\
\hline Opostegidae sp. 1 & $\mathrm{~F}$ & - & - & Acd & $\mathrm{N} / \mathrm{D}$ \\
\hline Tortricidae sp. 1 & $\mathrm{~F}$ & Acd & $\mathrm{N} / \mathrm{D}$ & - & - \\
\hline \multicolumn{6}{|l|}{ Insecta, Hemiptera, Hebriidae } \\
\hline Hebrus & $P$ & Acd & $\mathrm{D}$ & - & - \\
\hline Merragata & $\mathrm{P}$ & Acd & $\mathrm{N} / \mathrm{D}$ & - & - \\
\hline \multicolumn{6}{|l|}{ Insecta, Hemiptera, Mesoveliidae } \\
\hline Mesovelia & $P$ & Acd & $\mathrm{D}$ & Acd & $\mathrm{N} / \mathrm{D}$ \\
\hline \multicolumn{6}{|l|}{ Insecta, Hemiptera, Naucoridae } \\
\hline Limnocoris & $P$ & Acs & $\mathrm{D}$ & Acd & $\mathrm{N} / \mathrm{D}$ \\
\hline Pyrrhocoridae & $\mathrm{P}$ & Aus & $\mathrm{N} / \mathrm{D}$ & Acd & $\mathrm{N} / \mathrm{D}$ \\
\hline \multicolumn{6}{|l|}{ Insecta, Hemiptera, Veliidae } \\
\hline Microvelia & $P$ & Acd & $\mathrm{D}$ & Acd & $\mathrm{D}$ \\
\hline Insecta, Odonata, Calopterygidae & & & & & \\
\hline Hetarina & $P$ & Acd & $\mathrm{N} / \mathrm{D}$ & - & $\mathrm{N} / \mathrm{D}$ \\
\hline Insecta, Odonata, Coenagrionidae & & & & & \\
\hline Argia & $\mathrm{P}$ & $\mathrm{C}$ & $\mathrm{D}$ & Acd & $\mathrm{N} / \mathrm{D}$ \\
\hline Telebasis & $\mathrm{P}$ & Acd & $\mathrm{N} / \mathrm{D}$ & - & - \\
\hline Enallegma & $P$ & Acd & N/D & Acd & $\mathrm{N} / \mathrm{D}$ \\
\hline Homeoura & $\mathrm{P}$ & Acd & $\mathrm{N} / \mathrm{D}$ & - & - \\
\hline Insecta, Odonata, Corduliidae & & & & & \\
\hline Neocordulia & $\mathrm{P}$ & Acd & $\mathrm{N} / \mathrm{D}$ & Acd & $\mathrm{N} / \mathrm{D}$ \\
\hline Insecta, Odonata, Megapodagrionidae & $P$ & Acd & $\mathrm{N} / \mathrm{D}$ & - & - \\
\hline Insecta, Odonata, Libellulidae & & & & & \\
\hline Brechmorhoga & $P$ & Acd & $\mathrm{D}$ & - & - \\
\hline Dasythemis & $P$ & Acd & $\mathrm{N} / \mathrm{D}$ & - & - \\
\hline Diastatops & $\mathrm{P}$ & Acd & $\mathrm{D}$ & - & - \\
\hline Dythelmis & $\mathrm{P}$ & Acd & $\mathrm{D}$ & - & - \\
\hline Elga & $\mathrm{P}$ & Acd & $\mathrm{D}$ & - & - \\
\hline Erythrodiplax & $P$ & Acd & $\mathrm{D}$ & - & - \\
\hline Idiataphe & $P$ & Acd & $\mathrm{N} / \mathrm{D}$ & - & - \\
\hline Macrothemis & $P$ & Acd & $\mathrm{D}$ & - & - \\
\hline Orthemis & $\mathrm{P}$ & Acd & $\mathrm{D}$ & - & - \\
\hline Insecta, Odonata, Protoneuridae & & & & & \\
\hline Epipleoneura & $P$ & Acd & $\mathrm{D}$ & Acd & $\mathrm{N} / \mathrm{D}$ \\
\hline Platystictidae & $\mathrm{P}$ & Acd & $\mathrm{N} / \mathrm{D}$ & - & - \\
\hline Insecta, Odonata, Perilestidae & & & & & \\
\hline Perilestes & $\mathrm{P}$ & Acd & $\mathrm{D}$ & - & - \\
\hline Insecta, Trichoptera, Leptoceridae & & & & & \\
\hline Nectopsyche & $\mathrm{C} / \mathrm{R}$ & - & - & Acd & $\mathrm{N} / \mathrm{D}$ \\
\hline Oecetis & $\mathrm{F}$ & Acd & $\mathrm{N} / \mathrm{D}$ & - & - \\
\hline Triplectides & $\mathrm{F}$ & Acs & $\mathrm{D}$ & Acd & $\mathrm{N} / \mathrm{D}$ \\
\hline Insecta, Trichoptera, Hidroptilidae & & & & & \\
\hline Neotrichia & $\mathrm{R}$ & Acd & $\mathrm{D}$ & Acd & $\mathrm{D}$ \\
\hline Oxyetrichia & $\mathrm{R}$ & Acd & $\mathrm{D}$ & Acd & $\mathrm{N} / \mathrm{D}$ \\
\hline Insecta, Trichoptera, Hydropsychidae & & & & & \\
\hline Smicridea & $\mathrm{C} / \mathrm{F}$ & Acd & $\mathrm{N} / \mathrm{D}$ & Acd & $\mathrm{N} / \mathrm{D}$ \\
\hline Insecta, Trichoptera, Philopotamidae & & & & & \\
\hline Chimarra & $\mathrm{C} / \mathrm{F}$ & Acd & $\mathrm{N} / \mathrm{D}$ & Acd & $\mathrm{N} / \mathrm{D}$ \\
\hline Insecta, Trichoptera, Polycentropodidae & & & & & \\
\hline Cernotina & $\mathrm{C} / \mathrm{F}$ & Acd & $\mathrm{D}$ & Acd & $\mathrm{N} / \mathrm{D}$ \\
\hline
\end{tabular}


Tab. III. Cont.

\begin{tabular}{|c|c|c|c|c|c|}
\hline \multirow{2}{*}{$\begin{array}{l}\text { Composição de táxons } \\
\text { Invertebrados bentônicos }\end{array}$} & \multirow[b]{2}{*}{ GTF } & \multicolumn{2}{|c|}{ Tratamento 1} & \multicolumn{2}{|c|}{ Tratamento 2} \\
\hline & & Const & Dom & Const & Dom \\
\hline Cyrnellus & $\mathrm{C} / \mathrm{F}$ & Acd & $\mathrm{D}$ & Acd & $\mathrm{N} / \mathrm{D}$ \\
\hline Macrosternum & $\mathrm{C} / \mathrm{F}$ & Acd & N/D & - & - \\
\hline Polyplectropus & $\mathrm{R}$ & Acd & $\mathrm{D}$ & Acd & $\mathrm{N} / \mathrm{D}$ \\
\hline Collembola, Entomobryomorpha & $\mathrm{C} / \mathrm{C}$ & Acd & $\mathrm{N} / \mathrm{D}$ & - & - \\
\hline Turbellaria & $\mathrm{P}$ & Acs & $\mathrm{D}$ & Acd & $\mathrm{N} / \mathrm{D}$ \\
\hline Gastropoda, Ancylidae & $\mathrm{R}$ & $\mathrm{C}$ & $\mathrm{D}$ & Acs & $\mathrm{D}$ \\
\hline Gastropoda, Amphullaridae & $\mathrm{R}$ & Acd & $\mathrm{D}$ & - & - \\
\hline Gastropoda, Planorbidae & $\mathrm{R}$ & $\mathrm{C}$ & $\mathrm{D}$ & Acs & $\mathrm{D}$ \\
\hline Gastropoda, Thiaridae & $\mathrm{R}$ & Acd & $\mathrm{N} / \mathrm{D}$ & - & - \\
\hline Hirudinea & $\mathrm{P}$ & Acd & $\mathrm{D}$ & Acd & $\mathrm{N} / \mathrm{D}$ \\
\hline Acari, Hydracarina* & $P$ & $\mathrm{C}$ & $\mathrm{D}$ & $\mathrm{C}$ & $\mathrm{D}$ \\
\hline Cladocera* & $\mathrm{F} / \mathrm{F}$ & $\mathrm{C}$ & $\mathrm{D}$ & $\mathrm{C}$ & $\mathrm{D}$ \\
\hline Oligochaeta, Enchytraeidae* & $\mathrm{C} / \mathrm{C}$ & $\mathrm{C}$ & $\mathrm{D}$ & $\mathrm{C}$ & $\mathrm{D}$ \\
\hline Oligochaeta, Naididae* & $\mathrm{C} / \mathrm{C}$ & $\mathrm{C}$ & $\mathrm{D}$ & Acs & $\mathrm{D}$ \\
\hline Ostracoda, Cyprididae* & $\mathrm{F} / \mathrm{F}$ & $\mathrm{C}$ & $\mathrm{D}$ & $\mathrm{C}$ & $\mathrm{D}$ \\
\hline Ostracoda, Cytheridae* & $\mathrm{F} / \mathrm{F}$ & $\mathrm{C}$ & $\mathrm{D}$ & $\mathrm{C}$ & $\mathrm{D}$ \\
\hline Ostracoda, Darwinulidae* & $\mathrm{F} / \mathrm{F}$ & $\mathrm{C}$ & $\mathrm{D}$ & $\mathrm{C}$ & $\mathrm{D}$ \\
\hline Nematoda* & $\mathrm{P}$ & Acs & $\mathrm{D}$ & Acs & $\mathrm{D}$ \\
\hline
\end{tabular}

Tab. IV. Percentual de Grupos tróficos funcionais (GTF) para os invertebrados bentônicos nos Tratamentos 1 e 2 (P, Predador; C/C, Coletor-Catador; C/F, Coletor-filtrador; C/R, Coletor-raspador; F, Fragmentador; R, Raspador; F/F, Filtrador-fitófago), encontrados em bolsas de folhiço expostas no riacho Saltinho de fevereiro a julho de 2013, na Reserva Biológica de Saltinho,Pernambuco.

\begin{tabular}{lccccccc}
\hline GTF & $\mathrm{P}$ & $\mathrm{C} / \mathrm{C}$ & $\mathrm{C} / \mathrm{F}$ & $\mathrm{C} / \mathrm{R}$ & $\mathrm{F}$ & $\mathrm{R}$ & $\mathrm{F} / \mathrm{F}$ \\
\hline Tratamento 1 & 31,3 & 4,6 & 6,9 & 0 & 4,8 & 6,7 & 45,6 \\
Tratamento 2 & 18,0 & 4,1 & 7,3 & 0,1 & 0,6 & 2,3 & 67,7 \\
\hline
\end{tabular}

(Trichoptera) (253 indivíduos), seguido de Polypedillum (Diptera, Chironomidae) (30 indivíduos). Os demais correspondem a 57 indivíduos, distribuídos entre Stenochironomus (Diptera, Chironomidae), Oecetis (Trichoptera, Leptoceridade), Parapoynx (Lepidoptera, Crambridae), Wartzoyphius (Ephemeroptera, Baetidae), Tortricidae (Lepidoptera) e Dixella (Diptera, Dixidae) encontrados no tratamento 1 . $\mathrm{O}$ tratamento 2 apresentou um total de 30 indivíduos fragmentadores, sendo 14 representantes de Stenochironomus (14 indivíduos), Polypedillum (10 indivíduos) e três indivíduos de Epipyropidae e Opostegidae (Lepidoptera) cada um.

\section{DISCUSSÃO}

As análises de perda de massa foliar de I. ingoides mostraram que houve maior decomposição nas bolsas de colonização do que nas bolsas de exclusão. As bolsas de exclusão, mesmo com malha diminuta, permitiram a entrada de organismos da meiofauna e de estágios e/ou instar iniciais da macrofauna (e.g., larvas de Chironomidae, Ephemeroptera, Lepidoptera, Trichoptera, larvas de Coleoptera, entre outros). Este resultado indica a participação dos organismos da macrofauna na aceleração da decomposição do material foliar nos três trechos do riacho. É evidente que o processo de aceleração da decomposição ocorra na presença de organismos maiores (macrofauna), porém a participação da meiofauna nas bolsas de colonização e de exclusão foi evidenciada, pois a maioria dos grupos da meiofauna foi constante, como Cyprididae, Cytheridae e Darwinullidae (Ostracoda).
Petersen \& Cummins (1974) e Graça et al. (2005) classificaram a decomposição foliar como lenta os detritos foliares que apresentarem 0 a $0,005 \mathrm{dia}^{-1}$, média os que tem uma taxa de 0,005 a 0,010 dia $^{-1}$ e rápida a taxa de decomposição foliar de $0,010 \mathrm{dia}^{-1}$ ou superior.

A velocidade de decomposição de $I$. ingoides foi em geral média, com $\mathrm{k}=-0,0004 \mathrm{a}-0,012$. Porém, em estudos realizados por ENCALADA et al. (2010) no Equador, foi verificado que nas bolsas de maior malha, aos 63 dias de exposição com folhas de Inga, tiveram uma biomassa remanescente de $40 \%$. CAPPS et al. (2011), ao trabalharem com I. edulis na Amazônia, encontraram também 40\%R. Nosso estudo mostra que a $\% \mathrm{R}$ de $I$. ingoides em riacho de $1^{\mathrm{a}}$ ordem na Mata Atlântica de Pernambuco apresentam valores superiores $25 \%$ R, chegando até a 3\%R. Mesmo considerada como velocidade média, o processo de decomposição é mais acelerado do que nos estudos da Amazônia e Equador. Evidenciar quais os fatores que atuam nesse processo, ainda, é difícil de analisar. Magee (1993) enfatiza que há fatores externos como (i) águas mais oxigenadas, (ii) valores altos e neutros de $\mathrm{pH}$, (iii) baixa latitude e elevação, (iv) biomassa reduzida de microorganismos e (v) a alta densidade de invertebrados, acelera a decomposição do material vegetal em ecossistemas aquáticos. Outro fator que pode influenciar na decomposição é a velocidade do fluxo da água, por aumentar a abrasão física da água com a folha, fragmentando mecanicamente a mesma (АвELHo, 2001). A composição química das folhas também exerce influência na decomposição. Koptur (1984) revelou que folhas de Inga apresentam compostos químicos antiherbivoria e as classificou como folhas com grande quantidade de fenóis. 
Relatou também que esse composto pode influenciar na escolha da planta pelos herbívoros.

$\mathrm{O}$ fator tempo também foi significativo para as análises do processo de decomposição, tanto para a perda de massa foliar quanto para a abundância dos organismos. À medida que o tempo decorre, há acréscimo na colonização e sucessão de organismos menores (estágios iniciais de insetos e organismos considerados mais frequentes na meiofauna, como ostracodos, ácaros hidracarinos e vermes oligoquetos), evidenciados pela saturação da curva de rarefação para ambos os tratamentos. $\mathrm{O}$ incremento de táxons foi ocorrendo ao longo do tempo, como evidenciado nas curvas de rarefação, indicando que à medida que a biomassa foliar se torna mais palatável, aumenta o número de organismos.

Dentre os organismos mais abundantes estão Diptera (Chironomidae) e Crustacea (Ostracoda), para os dois tratamentos. Carvalho \& Uieda (2004), KikUChi \& Uieda (2005) e BatTistoni et al. (2010) relataram que Chironomidae é a família mais comumente encontrada, por ser cosmopolita e com uma grande diversidade de espécies, habitats e hábitos alimentares. Segundo Armitage (2005), Chironomidae domina as comunidades de insetos aquáticos em abundância e riqueza. NiN et al. (2009) encontraram valores superiores que $50 \%$ de representatividade de Chironomidae em riacho de altitude $1^{\text {a }}$ ordem no sul do Brasil, assim como BATTistToni et al. (2010) e Remor et al. (2013). Os quironomídeos apresentaram 59,2\% de coletores, seguido por fragmentador $(22,9 \%)$ e predador $(17,9 \%)$ nesse estudo. Em sua maioria foram considerados como Acidental, quanto à constância, e não dominantes. Isto se deve à alta diversidade de espécies de Chironomidae (Silva et al., 2007, 2009; Stenert et al., 2012).

Ostracoda foi o segundo táxon com maior abundância em ambos os tratamentos. Eles são comumente encontrados em ambientes dulciaquícolas, vivendo em diferentes tipos de hábitats, associados à vegetação, a macrófitas e ao sedimento (Albertoni \& WÜRDIG, 1996; PetKovski et al., 2002; LORENSCHAT \& SchWALB, 2013). Os resultados desse estudo corroboram com aquele realizado por CUNHA et al. (2014), que registraram maior predominância de DipteraChironomidae, Ostracoda e Coleoptera. CunHA et al. (2014) verificaram que os grupos predominantes na calha do riacho Saltinho (Chironomidae, Ostracoda e Coleoptera) apresentam hábito alimentar detritívoro e predador com variação em suas abundâncias devido às variáveis estruturais (substrato) e limnológicas (condutividade, oxigênio dissolvido e turbidez) do ecossistema. Em nosso estudo, Coleoptera apresentou-se como Acidental e não dominante. Em sua maioria os coleópteros neste ambiente são predadores. Somente Paracymus pertence ao grupo trófico funcional coletor-catador e Mycroceylloepus e Noelmis que são raspadores.

Os grupos tróficos funcionais observados nos detritos foliares de I. ingoides foram filtrador, seguido de predadores, coletores, raspador e fragmentador). Nessimian et al. (1999) e Henriques-Oliveira et al.
(2003) argumentam que muitos quironomideos não são restritos a um único hábito alimentar. Ostracodos podem ser considerados tanto filtradores, como em alguns casos coletores e fragmentadores. BARBOLA et al. (2011) registrou para o rio Pintangui no Paraná, como grupo trófico funcional predominante, os predadores, com mais de $40 \%$ de todos os organismos coletados. Essas configurações podem variar dependendo do tipo de recurso alimentar disponível nos trechos analisados. Não obstante, a escassez dos fragmentadores encontrados em regiões tropicais tem sido relatada (BOYERo et al., 2011)

É provável que os grupos tróficos variem com maior amplitude entre as latitudes. Porém, em regiões tropicais, a presença de variáveis de menor abrangência, como a diversidade do substrato (banco de folhas), tamanho e forma do corpo d'água, vazão, profundidade, entre outras, podem variar entre as regiões do Brasil. ARdón \& Pringle (2008) observaram que em riachos tropicais há predominância de grupos tróficos dos coletores. Neste estudo, o grupo trófico funcional predominante foi filtrador-fitófago, devido à elevada abundância de Ostracoda. $\mathrm{O}$ alto percentual de predadores pode ter sido influenciado pelo tempo de incubação das folhas, resultando em uma sucessão de grupos tróficos funcionais, pois ao decorrer do experimento os macroinvertebrados dos demais níveis tróficos tendem a incorporar a biomassa foliar, sendo repassada aos níveis tróficos superiores (predadores).

Os principais fatores que influenciam a abundância dos macroinvertebrados fragmentadores nos riachos tropicais são a temperatura e diversidade do banco de folhas oriundos da mata ciliar, não estando relacionados com a dureza das folhas, segundo Boyero et al. (2011). Com temperaturas mais elevadas o material foliar é atingido mais rapidamente pela atividade microbiana, tornando as folhas mais palatáveis, aos coletores, que se alimentam de material associados às folhas. BOYERo et al. (2011) associaram a escassez de fragmentadores nas regiões tropicais à baixa qualidade nutricional das folhas.

Os fragmentadores ocorreram em baixa frequência no riacho Saltinho, sendo os tricópteros considerados como fragmentadores verdadeiros (OliveIRA \& BisPo, 2001; Gonsales et al., 2002; Pes, 2002). Triplectides (Trichoptera, Leptoceridae) ocorreu com maior abundância entre os demais fragmentadores, seguido de Polypedillum (Chironomidae). Triplectides utiliza partes de galhos como casulos e se alimenta exclusivamente de folhas. SiLva et al. (2009), em estudos realizados no Centro-Oeste de São Paulo, mencionaram Triplectides, Polypedillum e Stenochironomus como fragmentadores. Foi evidenciada a influencia da macrofauna bentônica na perda de biomassa foliar acelerando a decomposição.

Agradecimentos. Ao ICMBIO e a Reserva Biológica de Saltinho pela autorização para execução da pesquisa. A Capes pela bolsa de mestrado ao autor-sênior. Ao Laboratório ARRE Água pela infraestrutura e depósito do material coletado. Ao Dr. Burkhard Sharf(Alemanha), por disponibilizar literatura referente à Ostracoda. A Lucas Lima (UFPE) pela confirmação na identificação de Ephemeroptera. 


\section{REFERÊNCIAS BIBLIOGRÁFICAS}

Aвelho, M. 2001. From litterfall to breakdown in streams: A review. The Scientific World 1:656-680.

Agra, U. M.; KLInK, J. M. \& Rodrigues, G. G. 2013. Monitoramento da piscicultura em reservatórios: uma abordagem ecológica. Revista Brasileira de Geografia Física 6(5):1457-1472.

Albertoni, E. F. \& Würdig, N. L. 1996. Comunidade de ostracodes associada à macrófitas aquáticas na Lagoa do Gentil, Tramandaí/ RS. Acta Limnologica Brasiliensia (8):103-104.

Anderson, J. M.; Proctor, J. \& Vallack, H. W. 1983. Ecological studies in four contrasting lowland rain forest in Gunung Mulu National Park, Sarawak. III. Decomposition processes and nutrient losses from leaf litter. Journal of Ecology 71(3):503-527.

Árdon, M. \& Pringle, C. M. 2008. Do secondary compounds inhibit microbial-and insect-mediated leaf breakdown in tropical rainforest stream, Costa Rica? Oecologia (155):311-323.

Armitage, D. 2005. Adaptive capacity and community-based natural resource management. Environmental Management 35(6):703-715.

Barbola, I. F.; Moraes, M. F. P. G.; Anazawa, T. M.; Nascimento, E. A.; Sepka, E. R.; Polegatto, C. M.; Milléo, J. \& Schühli, G. S. 2011. Avaliação da comunidade de macroinvertebrados aquáticos como ferramenta para o monitoramento de um reservatório na bacia do rio Pitangui, Paraná, Brasil. Iheringia, Série Zoologia 101(1-2):15-23.

Barbosa, A. F. \& Rodrigues, G. G. 2007. Variação da taxa de decomposição foliar de Ocotea puberula (Rich.) Nees ao longo da bacia hidrográfica Lajeado Grande, RS. Revista Brasileira de Biociências 5(1):906-908.

Battistoni, D.; Favassa, C. A.; Triques, R.; Barp, E. A. \& Rodrigues, G. G. 2010. Composição faunística de macroinvertebrados bentônicos ocorrentes na parte baixa do Rio Jacutinga, Concórdia, SC. Ágora: Revista de Divulgação Científica 17(1):20-31.

Benfield, E. F. 1996. Leaf breakdown in stream ecosystems. In: Hauer, F. R. \& Lambert, G. A. eds. Methods in stream Ecology. San Diego, Academic Press. 674p.

Benfield, E. F.; Paul, R. W. J. \& Webster, J. R. 1979. Influence of exposure technique on leaf breakdown rates in streams. Oikos (33):386-391.

Bodenheimer, F. S. 1938. Problems of animal ecology. Oxford, Oxford University Press. 179p.

Borkent, A. \& Spinelli, G. R. 2007. Neotropical Ceratopogonidae (Diptera: Insecta). In: Adis, J.; Arias, J. R.; RuedA-Delgado, G. \& Wantzen, K. M. eds. Aquatic Biodiversity in Latin America (ABLA). v. 4. Sofia-Moscow, Pensoft. 198p.

Boyero, L.; Pearson, R. G.; Dudgeon, D.; Graça, M. A. F.; Gessner, M. O.; Albarino, R. J.; Ferreira, V.; Yule, C. M.; Boulton, A. J.; Arunachalan, M.; Callisto, M.; Chauvet, E.; Ramirez, A.; Chará. J.; Moretti, M. S.; Júnior, J. F. G.; Helson, J. E.; CharÁ-Serna, A. M.; Encalada, A. C.; Davids, J. N.; Cornejo, A.; Li, A. O .Y.; Buria, L. M.; Villanueva, V. D.; Zuniga, M. C. \& Pringle, C. M. 2011. Global distribution of a key trophic guild contrasts with common latitudinal diversity patterns. Ecology 92(9):1839-1848.

Brower, J. E. \& Zarr, J. H. 1984. Field \& laboratory methods for general Ecology. 2ed. Iowa, W. C. Brown Company. 226p.

Capps, K. A.; Graça, M. A. S.; Encalada, A. C. \& Flecker, A. S. 2011. Leaf-litter decomposition across three flooding regimes in a seasonally flooded Amazonian watershed. Journal of Tropical Ecology (27):205-210.

Carvalho, E. M. \& UiEDA, V. S. 2004. Colonização por macroinvertebrados bentônicos em substrato artificial e natural em um riacho da Serra da Itatinga, São Paulo, Brasil. Revista Brasileira de Zoologia 21(2):287-293.

Cummins, K. W. 1973. Trophic relations of aquatic insects. Annual Review of Entomology 18(1):183-206.

Cunha, J. C. S.; Barros Filho, R. G.; Silva, R. P.; Santos, I. G. A. \& Rodrigues, G. G. 2014. Benthic macrofauna and the limological parameters of a first-order stream in Atlantic Forest of Brazilian Northeast. Acta Limnologica Brasiliensia 26(1):26-34.

Domínguez, E. \& Fernández, H. 2009. Macroinvertebrados Bentónicos Sudamericanos: Sistemática y Biología. Tucumán, Fundación Miguel Lillo. 656p.
Domínguez, E.; Molineri, C.; Pescador, M. L.; Hubbard, M. D. \& Nieto, C. 2006. Ephemeroptera of South America. In: AdIS, J; ArIAS, J. R.; Rueda-Delgado, G. \& Wantzen, K. M. eds. Aquatic Biodiversity of Latin America. v. 2, Sofia-Moscow, Pensoft. 642p.

Encalada, A. C.; Calles, J.; Ferreira, V.; Canhoto, C. N. \& Graça, M. A. S. 2010. Riparian land use and the relationship between the benthos and litter decomposition in tropical montane streams. Freshwater Biology (55):1719-1733.

Epler, J. H. 2010. The Water Beetles of Florida. Tallahasse, Florida Department of Environmental Protection. 414p.

Gonsales, E. L.; Coelho, F. M.; Romero, G. Q.; Santos, J. C. \& UeharaPrado, M. 2002. Seleção de habitat por invertebrados aquáticos fragmentadores em um Igarapé da Amazônia Central. Manaus, INPA. 55p.

GraçA, M. A. S; Bärlocher, F. \& Gessner, M. O. 2005. ed. Methods to study litter decomposition: a practical guide. Dordrecht, Springer. $313 \mathrm{p}$.

Hamada, N.; Nessimian, J. L. \& Querino, R. B. 2014. Insetos aquáticos na Amazônia Brasileira: taxonomia, biologia e ecologia. Manaus, INPA. 724p.

Henriques-Oliveira, A. L.; Nessimian, J. L. \& Dorvillé, L. F. M. 2003. Feeding habits of chironomid larvae (Insecta: Diptera) from a stream in the Floresta da Tijuca, Rio de Janeiro, Brazil. Brazilian Journal of Biology 63(2):269-281.

Lorenschat, J. \& Schwalb, A. 2013. Autecology of the extant ostracod fauna of Lake Ohrid and adjacent waters - A key to paleoenvironmental reconstruction. Belgian Journal of Zoology 143(1):42-68.

KIIUUCHI, R. M. \& UiEDA, V. S. 2005. Composição e distribuição dos macroinvertebrados em diferentes substratos de fundo de um riacho no município de Itatinga, São Paulo, Brasil. Entomologia y Vectores 12(2):193-231

Koptur, S. 1984. Experimental evidence for defense of Inga (Mimosoidae) sampling by Ants. Ecology (65):1787-1793.

Magee, P. A. 1993. Detrital accumulation and processing in wetlands. Waterfowl Management Handbook, Fish and Wildlife Leaflet 13(3): $1-7$.

Merritt, R. W.; Cummins, K. W. \& Burton, T. M. 1984. The role of aquatic insects in the processing and cycling of nutrients. Canadian Journal of Fisheries and Aquatic Sciences (43):101-113.

Mugnai, R.; Nessimian, J. L \& Baptista, D. F. 2010. Manual de identificação de macroinvertebrados aquáticos do Estado do Rio de Janeiro. Rio de Janeiro, Livros Técnicos Editora. 174p.

Nessimian, J. L.; SAnseverino, A. M. \& Oliveira, A. L. H. 1999. Relações tróficas de larvas de Chironomidae (Diptera) e sua importância na rede alimentar em um brejo no litoral do Estado do Rio de Janeiro. Revista Brasileira de Entomologia 43(1/2):47-53.

Nin, C. S.; Ruppenthal, E. L. \& Rodrigues, G. G. 2009. Produção de folhiço e fauna associada de macroinvertebrados aquáticos em curso d'água de cabeceira em floresta ombrófila do Estado do Rio Grande do Sul, Brasil. Acta Scientiarum, Biological Sciences 31(3):263-271.

OliveirA, L. G. \& Bispo, P. C. 2001. Ecologia de comunidades das larvas de Trichoptera Kirby (Insecta) em dois córregos de primeira ordem da Serra dos Pireneus, Pirenópolis, Goiás, Brasil. Revista Brasileira de Zoologia 18(4):1245-1252.

Passos, M. I. S.; Nessimian, J. L. \& Ferreira-Junior, N. 2007. Chave para identificação dos gêneros de Elmidae (Coleoptera) ocorrentes no Estado do Rio de Janeiro, Brasil. Revista Brasileira de Entomologia 51(1):42-53.

Pes, A. M. O. 2002. Categorias funcionais de alimentação de macroinvertebrados em igarapés da Reserva do km 41, Amazônia Central. Curso de Campo Ecologia da Floresta Amazônica, p. 115-116.

Petersen, R. C. \& Cummins, K.W. 1974. Leaf processing in a woodland stream. Freshwater Biology 4(4):343-368.

Petkovski, T. K.; Scharf, B. \& Keyser, D. 2002. New and little known species of the genus Candona (Crustacea, Ostracoda) from Macedonia and other Balkan areas. Limnologica (32):114-130.

Remor, M. B.; Hermoso, M.; Sgarbi, L. F.; Prestes, T. M. V. \& CÂmara, C. D. 2013. Qualidade da água do Rio das Pedras utilizando macroinvertebrados bentônicos como bioindicadores. Cultivando o Saber 6(2):97-111. 
Ruppenthal, E. L.; Nin, C. S. \& Rodrigues, G. G. 2007. A mata ciliar/curso d'água é um ecossistema único? Revista Brasileira de Biociências 5(1):861-863.

Silva, F. L.; Moreira, D. C.; Ruiz, S. S. \& Bochini, G. L. 2007. Avaliação da importância da unidade de conservação na preservação da diversidade de Chironomidae (Insecta: Diptera) no córrego Vargem Limpa, Bauru, Estado de São Paulo. Acta Scientiarum, Biological Sciences 29(4):401-405.

Silva, F. L.; Pauleto, G. M.; Talamoni, J. L. B. \& Ruiz, S. S. 2009. Categorização funcional trófica das comunidades de macroinvertebrados de dois reservatórios na região Centro-Oeste do Estado de São Paulo, Brasil. Acta Scientiarum, Biological Sciences 31(1):73-78.
Stenert, C.; Maltchik, L. \& Rocha, O. 2012. Diversidade de invertebrados aquáticos em arrozais no Sul do Brasil. Neotropical Biology and Conservation 7(1):67-77.

Trivinho- Strixino, S. 2011. Larvas de Chironomidae. Guia de Identificação. São Carlos, UFSCar, Departamento de Hidrobiologia, Lab. Entomologia Aquática. 371p.

Webster, J. R. \& Benfield, E. F. 1986. Vascular plant breakdown in freshwater ecosystems. Annual Review of Ecology and Systematics 17:567-594. 